\title{
The effect of fixation point on the appearance of rectilinearity*
}

\author{
LUBOMIR S. PRYTULAK† \\ University of Western Ontario, London, Ontario, Canada N6A $3 K 7$
}

\begin{abstract}
Data from two experiments indicate that (a) within $3.5 \mathrm{deg}$ visual angle of the fixation point, an eccentrically fixated line and dot appear rectilinear when they are objectively convex toward the fixation point; (b) beyond 3.5 deg, they appear rectilinear when they are objectively concave; (c) the latter effect is not an artifact of the use of stationary fixation points; and (d) the addition of an extraneous line to the stimulus line so as to form a 90 -deg angle acts as if to reduce the apparent size of the angle, but does not alter fixation point effects. The data were interpreted as supporting (a) the hypothesis that the stimulus line was more sensitive to illusory distortion than the dot, and (b) the ID (increasing-decreasing) model of the visual field - an inner area of increasing concentric contraction (stimuli appear to lie nearex the fixation point to a degree which increases with distance from the fixation point) is bounded by an outer area of decreasing concentric contraction.
\end{abstract}

The recognition that fixation point affects apparent tilt is not new: Helmholtz (1920, pp. 178-185), for example, described numerous commonplace situations in which the effect can be observed. The illustration in Fig. 1 is prototypical-when the middle dot is fixated, the three collinear dots appear collinear; when either $\mathrm{X}$ is fixated, however, the dots appear concave toward the fixated $X$. Best results will be obtained with page and head vertical.

In the present paper, this phenomenon is conceptualized, following Helmholtz (1920, p. 185), as "The apparent contraction of dimensions near the periphery that extend radially from the ... point of fixation." That is to say, the visual field is hypothesized to contract toward the fixation point and the contraction to increase with distance from the fixation point. This contraction is referred to below as "increasing concentric contraction" or merely "increasing contraction." Applied to Fig. 1, the increasing contraction model implies that because the end dots are farther from either $\mathrm{X}$ than the middle dot, they contract more, thereby producing the impression of curvature. Any eccentrically fixated straight line, therefore, is predicted to appear concave toward the fixation point and any eccentrically fixated line which appears straight must in actuality be convex. As the contraction is hypothesized to be concentric towards the fixation point, lines which are collinear with the fixation point may exhibit distortions of apparent length, but are predicted to exhibit none of apparent tilt or curvature.

During the five decades since Helmholtz's (1920) discussion of this phenomenon, it has been neglected: the contemporary psychological literature contains a single paper on the subject (Zając, 1957), and it merely replicates the basic phenomenon. This neglect is unfortunate for two reasons. First, fixation point

*This research was supported by Grant APA 390 from the National Research Council of Canada.

tThanks are due to Delbert A. Brodie for assistance in gathering the data. produces large, replicable, yet nonobvious, effects. If magnitude, stability, and lack of recognition of a phenomenon measure its importance to an understanding of the visual system, then fixation point effects are important.

Second, and more weighty, the disregard of fixation point as an experimental manipulation has gone hand in hand with, and perhaps encouraged, its disregard as an extraneous variable in need of control. In studies of apparent curvature or apparent tilt, the rule has been to let $\mathrm{S}$ fix his gaze where he will. With fixation point uncontrolled, however, one must face the possibility that E's acknowledged manipulation changed S's preferred fixation point and that the change in fixation point, in turn, contaminated the results. Undoubtedly, some data will eventually prove to be much the same whether fixation point is controlled or not. Just as surely, however, other data will be radically different. It is our inability to foretell into which of these categories any particular datum, gathered with inadequate attention to fixation point, will eventually be sorted that must make us digest it warily.

\section{$x$}

$x$

Fig. 1. Demonstration of an effect of fixation point. 


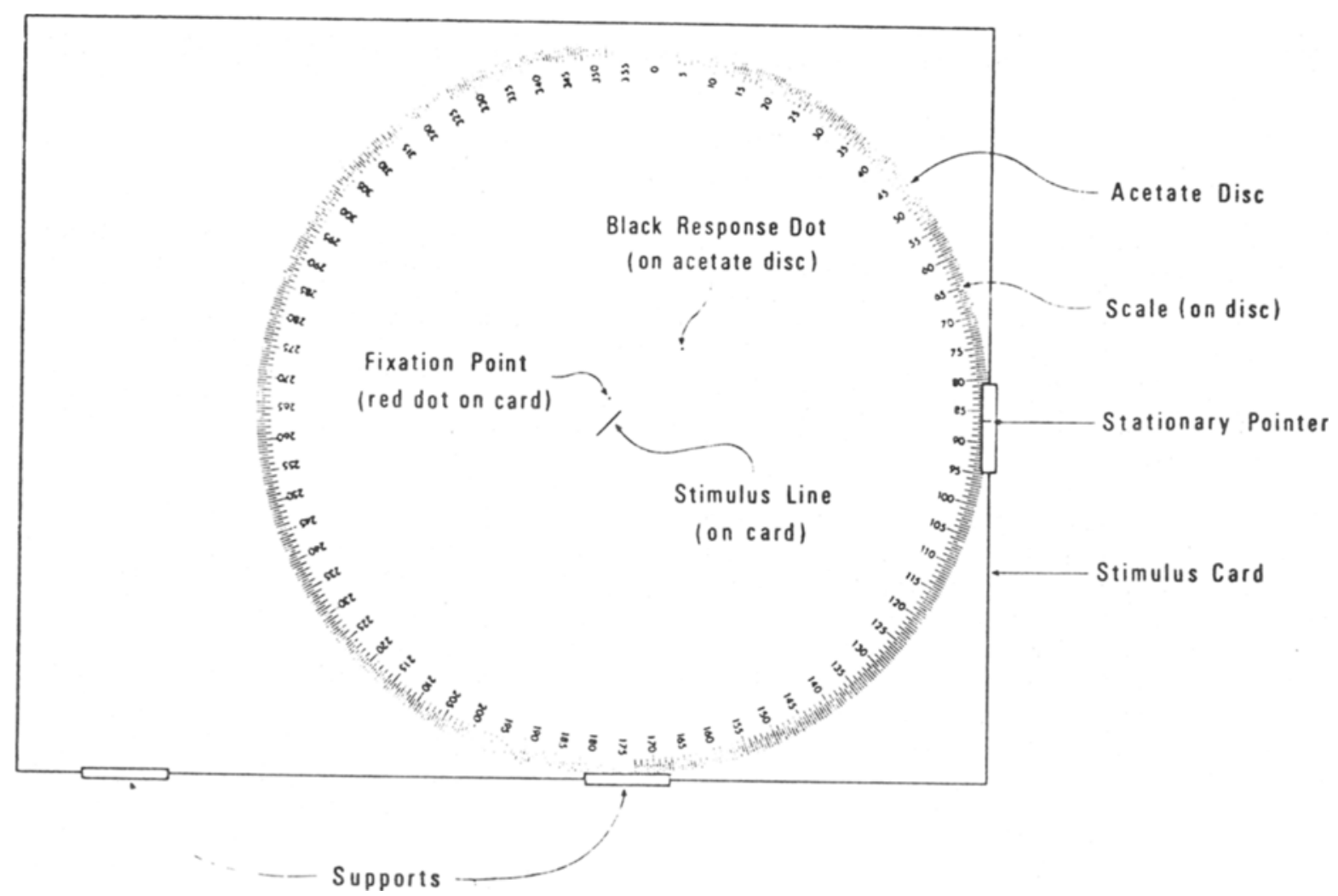

Fig. 2. Apparatus.

\section{GENERAL METHOD}

The $S s$ were presented with a stimulus line, a fixation point, and a movable dot. Their task was to set the dot so that it appeared collinear with the line while looking at the fixation point. The chief independent variable was the location of the fixation point. The dependent variable was the discrepancy between S's setting and a truly collinear setting.

\section{Apparatus and Procedure}

The working part of the apparatus is drawn to scale in Fig. 2. A black stimulus line $(1 \mathrm{~cm}$ long and $0.2 \mathrm{~mm}$ thick) and fixation point (a 0.3 -mm-diam red dot) are shown on a $21.6 \times 27.9 \mathrm{~cm}$ stimulus card. (A stimulus line tilted $45 \mathrm{deg}$ clockwise to the vertical as in Fig. 2. will be referred to as a 45 -deg stimulus line and one tilted $45 \mathrm{deg}$ counterclockwise to the vertical will be referred to as a 315 -deg stimulus line.)

The stimulus card rests inside two lower supports and is pulled snugly into a third support on the right. An acetate disc $(21.1 \mathrm{~cm}$ in diam and $0.19 \mathrm{~mm}$ thick) also rests inside two of the supports. On the disc is a black response dot $(0.3 \mathrm{~mm}$ in diam) and a 360 -deg scale printed in red so as to reduce its visibility in peripheral vision. When pressed with the fingers on its lower-right edge, the disc could be rotated so as to maintain contact with its supports at all times. As each stimulus line was drawn so that its "neartip" (the tip nearest the response dot) fell at the center of the disc, rotating the disc moved the response dot in a circle (radius $2.5 \mathrm{~cm}$ ) around the neartip.

While maintaining fixation of a given point. S would begin by moving the response dot in a few large-amplitude excursions bracketing the region in which the final setting would be made, then continued with back-and-forth movements of decreasing amplitude to a point where a few very small excursions were terminated by resting the dot in its final position. This gradual zeroing-in was aimed at reducing the effects of direction of approach and at making unnecessary the ascending and descending settings that would otherwise be required. After each setting, $S$ wrote the scale number (to the nearest half degree) that lay opposite the stationary pointer on the right-hand support, pulled out the stimulus card, located the card number on its back, and wrote the card number beside the scale reading.

The part of the apparatus shown in Fig. 2 was mounted on a $61 \mathrm{~cm}$ high $\times 76 \mathrm{~cm}$ wide board painted matte white and tilted back $15 \mathrm{deg}$ from the vertical. As the center of the stimulus display was 15 deg below S's horizontal line of sight, however. the display did lie in the frontoparallel plane-a chinrest mounted in front of the apparatus placed S's eyes at $45-\mathrm{cm}$ viewing distance and on either side of a perpendicular rising from the center of the display. The board was illuminated by two shielded incandescent bulbs, one $63 \mathrm{~cm}$ to either side of S's head, and also by two shielded fluorescent "warm white" bulbs $38 \mathrm{~cm}$ above and slightly forward of the center of the display. Illuminance from the stimulus card was $880 \mathrm{~cd} / \mathrm{m}^{2}$.

The deck of stimulus cards in each experiment was reordered between Ss by means of shuffling as well as by dealing the cards in haphazard order into separate piles, then picking the piles up in haphazard order.

\section{Location of the Fixation Point}

In order to be able to refer to fixation points in various parts of the display, a rectangular coordinate system is used: the y axis is placed along the stimulus line (and is, therefore, referred to as the stimulus axis); the $x$ axis passes through the neartip of the stimulus line. A fixation point's $\mathrm{x}$ coordinate is referred to as its eccentricity and its $y$ coordinate as its elevarion. Thus. when $S$ fixates the neartip. his fixation has an eccentricity of $0 \mathrm{~mm}$ and 
an elevation of $0 \mathrm{~mm}$. When he fixates the tip opposite the neartip. he fixates eccentricity $0 \mathrm{~mm}$, elevation $-10 \mathrm{~mm}$. Thus, elevation is positive for points lying close to the response dot, is zero for points opposite the neartip, and is negative for points still farther from the response dot. Eccentricity, on the other hand, is considered to be positive for points above the stimulus axis and negative for points below. When $S$ fixates a point $10 \mathrm{~mm}$ to the lower right of the neartip of a 45-deg stimulus line, therefore, his fixation has an eccentricity of $-10 \mathrm{~mm}$ and an elevation of $0 \mathrm{~mm}$.

\section{The Dependent Variable, $\theta$}

The dependent variable, $\theta$, is the difference between $S$ 's reading and the reading corresponding to an accurate collinear setting-that is, it is the angle between the stimulus axis and an imaginary line from the neartip to the response dot. In the graphic presentation of data (Figs. 3 and 4), $\theta$ was attributed a positive sign when $S$ placed the response dot too high, and a negative sign when he placed it too low. Also, when $\theta$ was zero, the response dot was said to have been placed collinearly. Placement of the response dot on the side of the stimulus axis contralateral to the fixation point was referred to as a contralateral response (CR), and placement on the side ipsilateral to the fixation point, as an ipsilateral response (IR).

It will be helpful to note that $C R$ indicates that an eccentrically fixated line and dot that are convex toward the fixation point appear rectilinear, and implies, therefore, that a rectilinear line and dot would appear concave. A CR, therefore, supports the notion of increasing concentric contraction of the peripheral visual field. An IR, on the other hand, implies that an eccentrically fixated rectilinear line and dot would appear convex and is compatible with the notion of a decreasing concentric contraction of the peripheral visual field. The possibility of decreasing concentric contraction, however, was not considered by either Helmholtz (1920) or Zając (1957).

\section{Subjects}

Students at the University of Western Ontario were paid to participate, usually in more than one experiment. As females were more cooperative and made fewer errors than males, recruitment of Ss was restricted almost entirely to females: 21 females and 2 males were tested in Experiment $I$, and 18 females and 1 male were tested in Experiment II. Three females and 1 male who participated in Experiment I also participated in Experiment II. An exception to the student category was the present author. who participated as an $S$ in Experiment $I$.

In order to reduce the effect of what appeared to be errors in dial reading (one reading might differ from all the others by about $100 \mathrm{deg}$, for example), outlying responses were tagged using Dixon and Massey's (1957) r ratio with $\alpha=.10$, and Ss were recalled and retested on the relevant cards. During the retest, Ss were given the same instructions as before, asked not to change their original modus operandi, and given no information on their previous responses.

\section{EXPERIMENT I}

\section{Method}

Thirty fixation points were tested on a 45 -deg stimulus line and 30 on a 315 -deg stimulus line. Fixation points assumed eccentricities of $0 . \pm 5$, and $\pm 11 \mathrm{~mm}$ at each of six elevations: 30 , $20,10,0,-5$, and $-10 \mathrm{~mm}$. With five eccentricities, six elevations, and two stimulus line orientations. 60 cards were presented in all. All fixation points except those falling on the stimulus line were marked with a red dot on the stimulus card. The three fixation points falling on the stimulus line at elevations of $0 .-5$, and $-10 \mathrm{~mm}$ wese indicated only by "lIXATE" and "NEARTIP." "MIDLINE." or "FARTIP"

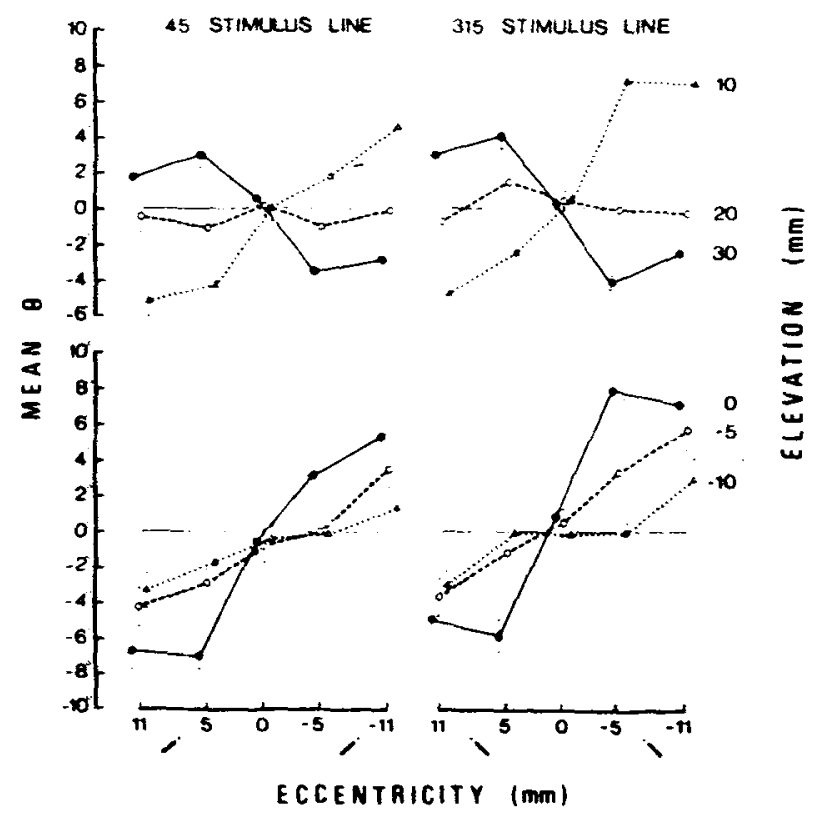

Fig. 3. Means with \pm 1 standard error of each mean, Experiment I. (Solid functions have been displaced slightly left, and dotted functions slightly right, to prevent crowding.)

printed in red (as were all special instructions) in the upper-left corner of the stimulus card.

\section{Results}

Means and intervals representing \pm 1 standard error of each mean are plotted in Fig. 3. The two graphs on the left present data from the 45-deg line, and the two graphs on the right from the 315-deg line. In the two upper graphs, the solid, dashed, and dotted functions present data from successively lower elevations: 30,20 , and $10 \mathrm{~mm}$. Similarly, in the two lower graphs, the solid. dashed, and dotted functions continue to present data from successively lower elevations: $0,-5$, and $-10 \mathrm{~mm}$.

Below each abscissa are nonscale representations of the stimulus line, the response dot, and hatching to indicate the side on which the fixation points at that region of the abscissa lay. Thus, along each abscissa, data from fixation points above the stimulus axis are plotted to the left, and from fixation points below the stimulus axis, to the right. In each graph, therefore, $C R$ would be indicated on the left by settings below the stimulus axis (negative $\theta$ ) and on the right by settings above the stimulus axis (positive $\theta$ )-in other words, by a positively sloped function lying in Quadrants I and III; IR, on the other hand, would be indicated by a negatively sloped function lying in Quadrants II and IV.

The following generalizations appear to fit the data. First, zero-eccentricity fixations along the length of the stimulus axis from 30 - to $-10 \cdot \mathrm{mm}$ elevation produced relatively accurate settings: none of the 12 mean $\theta \mathrm{s}$ exceeded an absolute value of $0.85 \mathrm{deg}$ (mean $\theta$ was $-0.85 \pm 0.89 \mathrm{deg}$ at $-5 \cdot \mathrm{mm}$ elevation of the $45 \cdot \mathrm{deg}$ line 
and was $0.85 \pm 0.60 \mathrm{deg}$ at $0-\mathrm{mm}$ elevation of the 315-deg line).

Next, at $30-\mathrm{mm}$ elevation. IR appeared for both 45 and 315-deg stimulus lines. As mentioned above. IR implies decreasing contraction-a finding which is surprising, because the demonstrations of Helmholtz (1920). Zajac (1957). or the one presented in Fig. 1 above imply only increasing contraction.

At $20-\mathrm{mm}$ elevation. settings are relatively accurate and eccentricity of viewing may be said to have had little effect, if any. The functions may be roughly characterized as $\theta=0$.

Progressing to the next-lower tier of fixation points, at $10-\mathrm{mm}$ elevation, an unmistakable CR has emerged: here is the increasing contraction that had been expected originally. At $0-\mathrm{mm}$ elevation, CR became even stronger (in $8 / 8$ comparisons). At $-5-\mathrm{mm}$ elevation, CR weakened ( $8 / 8$ comparisons) from its most powerful manifestation at $0 \cdot \mathrm{mm}$ elevation, and weakened yet again ( $8 / 8$ comparisons) at -10 -mm elevation.

The magnitude of the illusions may be appreciated by computing the range of mean settings for alternative fixation points at given elevations: $\mathrm{CR}$ at $0-\mathrm{mm}$ elevation produced mean $\theta$ s ranging over $12.41 \mathrm{deg}$ for the 45 -deg line and $13.76 \mathrm{deg}$ for the $315 \mathrm{deg}$ line. Similarly, IR at $30-\mathrm{mm}$ elevation produced mean $\theta \mathrm{s}$ ranging over $6.50 \mathrm{deg}$ for the 45-deg line and $8.11 \mathrm{deg}$ for the 315-deg line. Within the eccentricities tested, then, CR at its strongest was almost twice the magnitude of IR at its strongest.

The eccentricity that produces maximal $\theta$ appears to depend on position along the stimulus axis-IR at $30-\mathrm{mm}$ elevation (4/4 comparisons) and $\mathrm{CR}$ at $0-\mathrm{mm}$ elevation (3/4 comparisons) were greater at an eccentricity of $5 \mathrm{~mm}$ than at one of $11 \mathrm{~mm}$. The CR opposite10-, -5-, and -10-mm elevation, however, was greater at $11-\mathrm{mm}$ eccentricity ( $11 / 12$ comparisons).

The key findings, then, are that, with sufficiently eccentric fixation, CR occurred at elevations of -10 to $10 \mathrm{~mm}$; at $20-\mathrm{mm}$ elevation, however, eccentricity had no effect, and at $30 . \mathrm{mm}$ elevation produced IR.

The key result of an analysis of variance was a highly significant Eccentricity by Elevation interaction, $F(20,440)=46.41, p<.01$. The nature of the interaction, furthermore, was such as to weaken the main effects of the component variables: the main effect of eccentricity was weakened by some of the functions having a negative or zero slope, but was significant, nevertheless, because the majority of the functions were positively sloped, $F(4,88)=36.27, p<.01$; the main effect of elevation, on the other hand, vanished entirely because the mean heights of the functions did not differ, $F(5,110)=1.56, p>.01$. Orientation was not significant, $F(1,22)=6.43, p>.01$. Of the remaining interactions, only one reached significance at the .01 level: the Eccentricity by Elevation by Orientation interaction, $F(20,440)=1.94, p<.01$; in view of the comparative smallness of the effect, however, it will not be discussed.

\section{Discussion}

First, the repated observation of illusions of startling magnitude-a 13.76-deg difference in mean response settings produced by a $10-\mathrm{mm}$ (1.27-deg visual angle) change in fixation points. for example-supports the claim that fixation point is a powerful manipulation and, therefore, particularly worthy of attention.

Second, although the observation of CR (implying increasing contraction) had been expected from the demonstrations of Helmholtz (1920) and Zając (1957), the appearance of IR (implying decreasing contraction) was unprecedented and suspected of being artifactual. It was entertained, for example, that $S$ might have used the red-dot fixation points to derive information on the true location of the stimulus axis-he might have come to realize that when a fixation point looked close to the stimulus axis, it really was on the axis, and when it looked above or below, it had to be above or below by one of two constant distances. When the fixation point lay in the vicinity of the response dot, therefore, it could help S place the response dot more accurately-but could it have also produced an artifactual IR?

Suppose that in using the fixation points as clues to the location of the stimulus axis, S made mistakes. Suppose, for example, that the nearer a fixation point was to the axis, the more likely $\mathbf{S}$ was to make the error of assuming that it lay on the axis. This error, of course, would only be possible when the fixation point was sufficiently distant from the neartip-which is the very location that produced IR. What data would this error generate? Quite simply, S's setting would follow the fixation point. When the fixation point lay on the axis, S's setting would be accurate, and when the fixation point lay above or below the axis, his setting would be too high or too low, respectively. In other words, settings would be ipsilateral. Also, the error of assuming that a fixation point lies on the stimulus axis should be less frequent at larger eccentricities; the artifactual IR, therefore, should be weaker at $11-\mathrm{mm}$ than at $5-\mathrm{mm}$ eccentricity. Data from Experiment I (Fig. 3) confirm this expectation in $4 / 4$ comparisons.

Can the above interpretation of IR be refuted? Experiment II made the attempt.

\section{EXPERIMENT II}

Experiment II attempted to prevent $S$ from erroneously assuming that a fixation point was any specific distance from the stimulus axis by putting the fixation point on the moving acetate disc. The main purpose of Experiment II, then, was to determine whether mobile fixation points, bearing no simple relation to the location of the stimulus axis, made IR disappear.

Experiment II also tested the possibility that fixation point effects are peculiar to single-line stimuli and, therefore, not relevant to more complex displays such as the classical illusion displays or the more complex 
displays presented as stimuli in other laboratory research. Specifically, Experiment II compared settings to a single stimulus line with settings to a stimulus line whose neartip was abutted by an extraneous line so as to form an angle. The ability to generalize to angles is particularly critical in view of their ubiquitous presence in the classical illusions of tilted line.

\section{Method}

One-centimeter lines at 45 and $315 \mathrm{deg}$ were presented alone or with a second $1-\mathrm{cm}$ line abutting their neartip so as to form a 90-deg angle having arms symmetrical about the vertical and apex upward. The Ss were instructed that when a single line was presented, they were to place the response dot on the line's upward extension, and when an angle was presented, to place the response dot on the upward extension of one of the arms of the angle-Ss knew which arm to use by the instructions, "DOT LEFT $\leftarrow, "$ indicating that the response dot should be placed on the upper extension of the 315-deg arm, or "DOT RIGHT $\rightarrow$," indicating that it should be placed on the upper extension of the 45-deg arm.

Three fixation points were tested at $0-\mathrm{mm}$ elevation-one at $0-\mathrm{mm}$ eccentricity, indicated by "FIXATE NEARTIP," and two at $6-\mathrm{mm}$ eccentricity, each indicated by a red dot on the stimulus card. Three more fixation points were tested at $25-\mathrm{mm}$ elevation-one the response dot, indicated by "FIXATE BLACK DOT," and two at 6-mm eccentricity from the response dot, each indicated by a red dot on a different acetate disc.

Overall, two stimulus-line orientations, presence or absence of an extraneous line, and six fixation points gave 24 different displays. These were duplicated to give a total of 48 stimulus cards. Each subdeck of 24 was reordered before presentation to each $S$, but the subdecks were not mixed together. They were, however, placed on top of one another in a single deck, and $S$ worked through the deck without being informed that the stimuli repeated themselves.

\section{Results}

For each $S$, the responses to identical conditions within first and second subdecks were averaged, giving 24 data points per S. An analysis of variance of these data produced a pattern resembling Experiment I. First, the Eccentricity by Elevation interaction was highly significant, $F(2,36)=86.47, p<.01$. This interaction, in turn, reduced the main effect of eccentricity, but not below significance $-F(2,36)=8.39, p<.01$-and wiped out the main effect of elevation altogether $-F(1,18)=$ $0.48, \mathrm{p}>.01$. As before, orientation was not significant, $\mathrm{F}(1,18)=7.51, \mathrm{p}>.01$.

Means (with \pm 1 standard error of each mean) are plotted in Fig. 4. Examining the single-line-stimulus data first (solid functions, Fig. 4), we note the following replicative effects: (a) relatively collinear settings for zero-eccentricity fixations, (b) IR for eccentric fixation at $25-\mathrm{mm}$ elevation, (c) CR for eccentric fixation at $0-\mathrm{mm}$ elevation, and (d) CR larger than IR. The persistence of a robust IR, then. permits us to reject the interpretation that in Experiment I. IR was an artifact of S's use of stationary fixation points to extract erroneous information on the location of the stimulus axis.

The new observation is that the addition of the

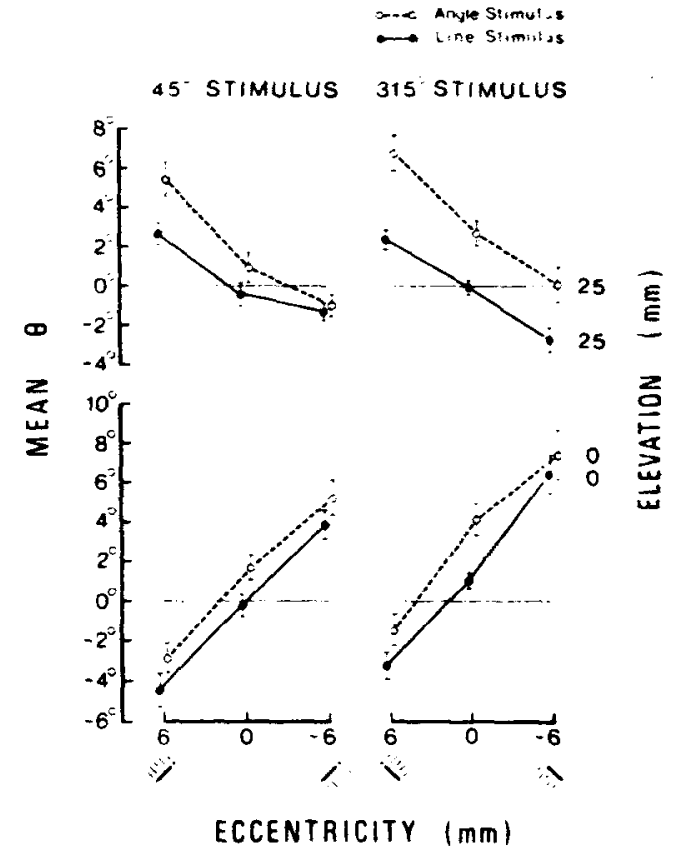

Fig. 4. Means with \pm 1 standard error of each mean, Experiment II. (Functions have been displaced laterally to prevent crowding.)

extraneous line raised settings in $12 / 12$ comparisons (dashed functions, Fig. 4) $-F(1,18)=26.45, p<.01$. This effect is compatible with the interpretation that a stimulus line forming an angle (compared to a stimulus line by itself) appears rotated toward the extraneous line as if to form a smaller angle. The effect of line, moreover, depended on eccentricity and on orientation: Line by Eccentricity, $F(2,36)=7.80, p<.01$; Line by Orientation, $F(1,18)=15.38, p<.01$. Although the differential effect of line is evident in Fig. 4 (as in the particularly large effect for fixation at $25-\mathrm{mm}$ elevation of the 315-deg stimulus line), it assumes no simple pattern, and in view of its comparatively small magnitude, will not be discussed in greater detail here. Although the Line by Eccentricity interaction indicates that the effect of fixation point changes upon the addition of the extraneous line, the effect is equally strong and qualitatively similar. This similarity and strength encourage the expectation that fixation point phenomena are not restricted merely to single-line stimuli, and are likely to be present during the inspection of more complex displays such as the classical geometrico-optical illusions.

A word of caution-the eccentricity of a stationary fixation point is its distance from the stimulus axis. but the eccentricity of a mobile fixation point is its distance from the response dot. Thus, a mobile fixation point $6 \mathrm{~mm}$ from the response dot is $6 \mathrm{~mm}$ from the stimulus axis only when a setting is collinear. When a setting is ipsilateral. however. eccentricity is greater than $6 \mathrm{~mm}$. and when contralateral. is less. It would have been 


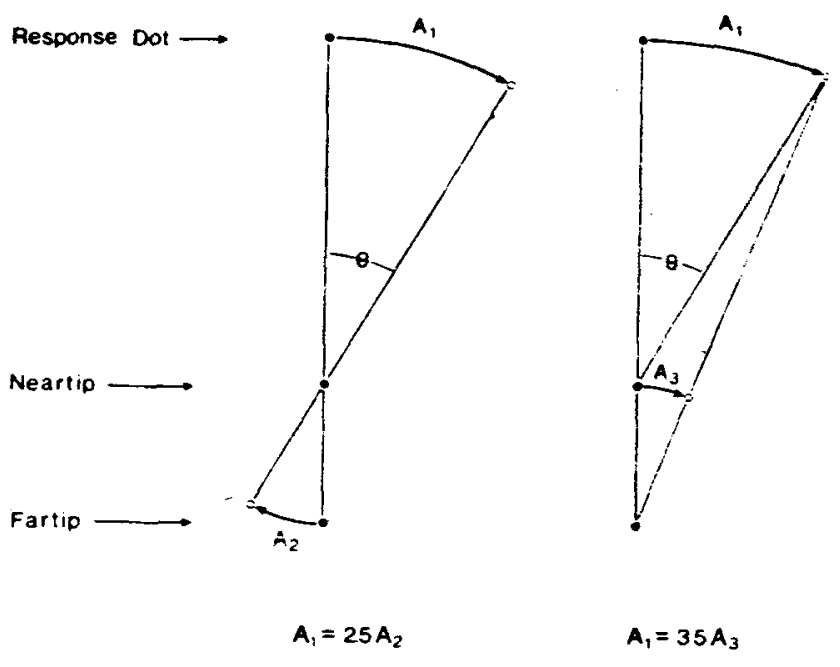

Fig. 5. Apparent displacement of the neartip or fartip of a short stimulus line affects $\theta$ more than an equivalent displacement of the response dot.

possible to plot mean $\theta \mathrm{s}$ from mobile-fixation-point conditions against a calculated mean eccentricity at S's final setting of the response dot. This alternative was not preferred, however, because it would have made only a small difference in the slopes of the functions, and none whatsoever in the conclusions.

\section{DISCUSSION}

It is hypothesized that the stimulus displays in Experiments I and II were more sensitive to illusory distortion in the area of the visual field occupied by the stimulus line than in the area occupied by the response dot-that is, whatever distortion was present in the region of the field occupied by the stimulus line weighed disproportionately in affecting $\theta$. This hypothesis is explained with the help of Fig. 5.

The illustrations in Fig. 5 assume a $1-\mathrm{cm}$ stimulus line and a $2.5-\mathrm{cm}$ response radius. On the left, the magnitude of illusory displacement (in a circular path around the neartip) required of the response dot to produce a given $\theta$ is 2.5 times that required of the fartip (the tip of the stimulus line farthest from the response dot). Similarly, on the right, the magnitude of displacement (in a circular path around the fartip) required of the response dot to produce a given $\theta$ is 3.5 times that required of the neartip. The exact value of each ratio, of course, depends on the locus of the illusory displacement, on the length of the stimulus line, and on the response radius. The conservative conclusion relied on here, however, is merely that when line length is shorter than response radius, settings to collinearity will be more sensitive to illusory displacement of the neartip or fartip than to an equal illusory displacement of the response dot.

Given the extra sensitivity of the stimulus line, the following model of the visual field fits the data: increasing contraction (indicated by CR) is characteriștic of an area within approximately $3.5 \mathrm{deg}$ visual angle of the fixation point, and decreasing contraction (indicated by IR) is characteristic of an area lying beyond $3.5 \mathrm{deg}$ visual angle of the fixation point. The use of this model to describe the findings of Experiment $I$ is shown in Fig. 6.

In each illustration in Fig. $6, \mathrm{X}$ marks the fixation point and the circle marks the border between the inner area of increasing contraction and the outer area of decreasing contraction. Above $\mathrm{A}$, the location in the visual field of stimuli fixated at 6 - and $11-\mathrm{mm}$ eccentricity at $-10-\mathrm{mm}$ elevation are shown. The stimulus lines fall within the area of increasing contraction, and the dots within the area of decreasing contraction. As the stimulus lines are more sensitive, CR should predominate, although it will be weakened by the dots' promotion of IR. Fixation at 0-mm elevation (B) places both lines and dots within the area of increasing contraction; maximal CR, therefore, should be observed. Fixation at $20-\mathrm{mm}$ elevation (C) places the dots in the region of $C R$ and the lines overlapping $C R$ and $I R$; the effects, therefore, could cancel. Fixation at $30-\mathrm{mm}$ elevation (D) places the dots in CR, but the more sensitive lines in IR; a weak IR, therefore, should predominate. The increasing-decreasing (ID) model, therefore, gives a nice fit to the fixation point effects reported above.

\section{Optical Distortion}

It is possible to account for increasing concentric contraction, and thus CR, by means of the optical properties of the eye (Davson, 1972, p. 616). An analogy will make this account clear.

If we position ourselves so that we are looking into a coffee cup, but from the side so that we can see only a part of the bottom, and if with a peashooter we shoot a

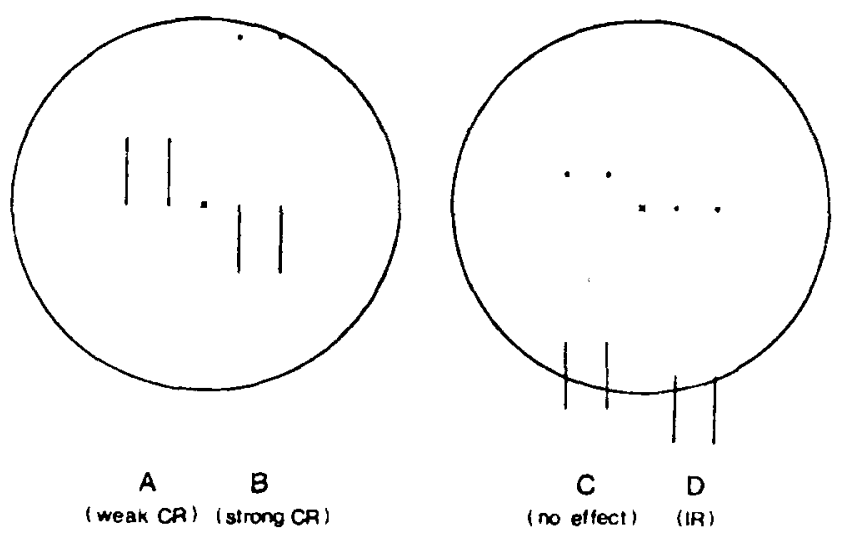

Fig. 6. The ID model applied to the stimulus displays presented in Experiment I. 
pea so as to hit the bottom, the pea will hit that part of the bottom lying farthest from us. Now, if the upper rim of the cup represents the edge of the pupil, and if the bottom of the cup represents the lens, and the pea is light entering the eye, we see that light from a peripheral source is directed away from the center of the lens and toward the contralateral edge. But, because the edge of the lens refracts more strongly than the center, the light from a peripheral source will end up being focused disproportionately close to the fovea-hence, increasing concentric contraction or CR. The resulting distortion is referred to as barrel distortion, because light from a rectangular grid passing through the system is projected on the retina in the shape of a barrel-that is, increasingly peripheral lines are projected as increasingly concave toward the fixation point.

A testable implication of this interpretation is that the magnitude of $C R$ should be inversely proportional to pupillary dilation: when the pupil is dilated, some of the light from the peripheral source is permitted to pass through the center of the lens, and even through the ipsilateral edge, which counteracts the effect of its passing through the contralateral edge. The experiments presented above, however, attempt to ascertain what the distortion is but not where it originates. That it originates in the optical system, then, is offered as a possibility, but not as one that is supported by the data to the exclusion of other possibilities.

At the same time, it must be recognized that optics provides a plausible interpretation for a portion of the data only-it can account for the CR observed within $3.5 \mathrm{deg}$ visual angle of the fixation point but not for the IR observed beyond 3.5 deg. Davson (1972, p. 617) points out that pin-cushion distortion (which is the same as decreasing concentric contraction and which would be manifested by IR) would be produced if the pupillary opening lay between the lens and the retina, but it does not seem likely that the IR observed in Experiments I and II was produced in this way. Furthermore, the effect of abutting an extraneous line to the stimulus line is even less amenable to an optical interpretation-the optical portion of the visual system seems to have no mechanism whereby the exceedingly small decrement in light which the extraneous line imposes on one part of the system could displace the image formed by light passing through a neighboring part. Clearly, physiological optics would have to be elaborated before it could account for more than a fraction of the data presented above.

\section{REFERENCES}

Davson, H. The physiology of the eye. (3rd ed.) New York: Academic Press, 1972.

Dixon, W. J., \& Massey, F. J., Jr. Introduction to statistical analysis. New York: McGraw-Hill, 1957.

Helmholtz, H. L. F. von. Handbuch der physiologischen Optik. Vol. III. (3rd ed.) Hamburg and Leipzig: Voss, 1920 [Republished in translation in J. P. C. Southall (Ed.), Helmholtz's treatise on physiological optics. Vol. III. Rochester, N.Y: Optical Society of America, 1925.]

Zajac, J. L. Some investigations on the so-called "geometrico optical illusions." Acta Psychologica, 1957, 13, 140-150.

(Received for publication March 23, 1973; revision received June 6,1973 .) 